\title{
L'autofiction dans Mes Mauvaises Pensées de Nina Bouraoui
}

\author{
DR. Dina Ahmed Mohamed Zaater
}

Maître de conférences au département de Français à la Faculté de Pédagogie d'Ain Shams 



\section{Résumé}

Le terme « autofiction » a produit de nombreuses théories et polémiques dans l'espace littéraire. Ce concept est un « genre nouveau » ou une « catégorie générique nouvelle » qui s'applique tout particulièrement à l'œuvre littéraire contemporaine comme l'affirme Philippe Gasparini. En lien avec cette notion, qui met en avant une combinaison d'éléments relevant d'une part de l'autobiographie et d'autre part, de la fiction, nous pouvons envisager "Mes mauvaises Pensées » de Nina Bouraoui comme une écriture autofictionnelle à travers l'application de dix attributs du concept doubrovskien d'après Gasparini.

Mots-clés : Récit autofictionnel. Nina Bouraoui. Serge Doubrovsky. Philippe Gasparini. Littérature francophone. الملضص : أسفر مصطلح "الخيال الذاتي" عن العديد من النظريات والنقاشات في

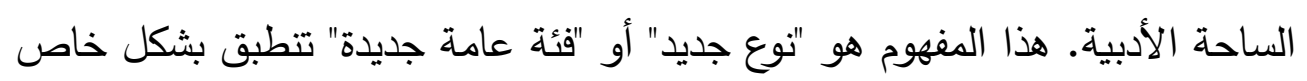

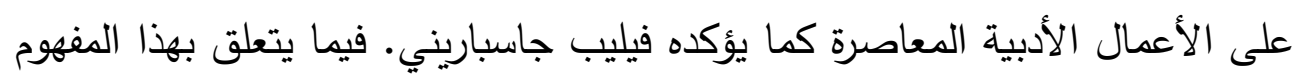

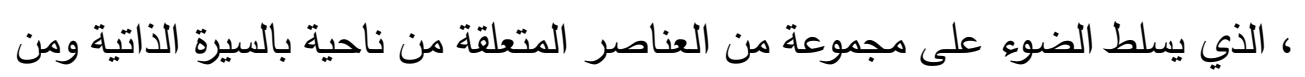

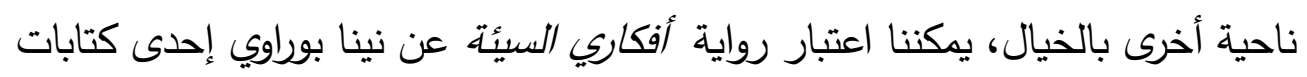
الخيال الذاتي و ذلك من خلال تطبيق عشر سمات لدفهوم دوبروفسكي عن الخيال الذاتي وفقا لجاسباريني.

الكلمات المفتاحية: نص خيال ذاتي، نينا بوراوي، سارج دوبروفسكي، فيليب جاسباريني. 
«Mon âme se dévore, je suis assiégée. Je porte quelqu'un à l'intérieur de ma tête, quelqu'un qui n'est plus moi ou qui serai un moi que j'aurais longtemps tenu, longtemps étouffé. » $\left(M M P^{1}\right.$, p.9)

Le terme « autofiction » a produit de nombreuses théories et polémiques dans l'espace littéraire. Ce concept a provoqué une hantise que Vincent Colonna a bien exprimée par ces mots :

«Un néologisme hante l'Empire des lettres, de l'Europe aux Amériques. Le mot hante les suppléments littéraires, les radios et les télévisions lettrées, les revues, les colloques, les études savantes ; hante l'écriture des jeunes comme de vieux écrivains ; et cette hantise contamine jusqu'au lecteur curieux, intrigué par ce terme protéiforme, tantôt label d'originalité, tantôt marque infamante, associé à des livres récents, plaqué sur des livres anciens. [...]. Depuis une quinzaine d'années, le nombre d'ouvrages décrits comme autofiction ou s'autoproclamant tels, ne cesse d'augmenter» (Colonna, 2004 : p.11).

${ }^{1}$ Nina BOURAOUI, Mes Mauvaises Pensées, Paris, éd. Stock, 2005. Ce neuvième récit de BOURAOUI a reçu le prix de Renaudot la même année de sa publication. Nous allons désormais utiliser l'abréviation $M M P$ pour désigner le récit en question, la pagination après le signe abréviatif. 
Dans son discours, Colonna met en lumière ce genre littéraire qui témoigne de la vivacité de la littérature. Bien qu'il y voie une tradition littéraire fort ancienne (voir Colonna, 1989 : p.11), Philippe Gasparini affirme que l'autofiction est un «genre nouveau» ou une «catégorie générique nouvelle » qui s'applique tout particulièrement à l'œuvre littéraire contemporaine (Gasparini, 2009).

En lien avec cette notion, pourrions-nous envisager «Mes Mauvaises Pensées » de Nina Bouraoui comme une écriture autofictionnelle ? Surtout que l'écrivaine nommée « experte en autofiction » (Jay, 2010) ne semble pas trop aimer ce terme : «C'est un mot qui me révulse un peu, car je ne le comprends pas » (JOURNET, 2011).

Alors, pour répondre à la question posée, notre démarche sera d'analyser les dix critères de l'autofiction selon Philippe Gasparini afin de déceler les rapports entre l'œuvre en question et la pratique de l'autofiction.

Or, il nous paraît nécessaire de commencer par un rappel théorique sur l'essence poétique de l'autofiction pour mieux juger de sa manifestation au sein du texte objet d'étude. 
L'autofiction et les débats théoriques : Historique et réception problématique du terme

Le néologisme "autofiction" est apparu sur la quatrième de couverture du premier roman de Serge Doubrovsky intitulé «Fils ». II est défini comme suit : " Fiction, de faits et d'évènements strictement réels. Fragments épars, morceaux dépareillés, tant qu'on veut : l'autofiction sera l'art d'accommoder les restes, d'avoir confié le langage d'une aventure à l'aventure du langage, hors sagesse et hors syntaxe du roman, traditionnel ou nouveau. Rencontre, fils des mots, allitérations, assonances, dissonance. » (Doubrovsky, 1977)

L'objectif de Doubrovsky était de remplir une "case aveugle" vide, laissée par Philippe Lejeune dans son ouvrage «Le Pacte autobiographique » où Lejeune (1995, pp.28, 31) niait la possibilité de coexistence d'un pacte autobiographique et romanesque dans une même œuvre.

Ce concept doubrovskien désigne donc une combinaison d'éléments relevant d'une part de l'autobiographie et d'autre part, de la fiction ou du roman dont la particularité réside en ce qu'il emprunte des caractéristiques de l'une pour les opposer à l'autre et vice-versa. II se distingue de l'autobiographie par le fait qu'il fait appel à la fiction de quoi il se différencie par son aspect 
factuel fondé sur les éléments réels et les faits historiques racontés.

En effet, le néologisme de Doubrovsky a suscité des réactions variées de la part des critiques littéraires. Certains, comme Gérard Genette, ont rejeté le terme, traitant les autofictions d' " autobiographies honteuses» (Genette, 1991 : p. 87). Marie Darrieussecq (1996, pp.369-380) présente son questionnement sur le "sérieux" du genre autofictionnel. Sébastien Hubier parle de « contradictions de l'autofiction qui, variante matoise de l'autobiographie, ne serait jamais qu'un genre indécis, hybride, conjointement fictionnel et autoréférentiel » (Hubier, 2003 : p.122).

D’autres modifient la définition de Doubrovsky en considérant l'autofiction comme une «fictionnalisation de soi » (Colonna, 1989 : p. 6), ce qui amène l'auteur de Fils à préciser sa définition à lui : «Ma conception de l'autofiction n'est pas celle de Vincent Colonna : "OEuvre littéraire par laquelle un écrivain s'invente une personnalité et une existence, tout en conservant son identité réelle (son véritable nom)." La personnalité et l'existence en question ici sont les miennes, et celles des personnes qui partagent ma vie» (Doubrovsky, 1993 : p. 212). 
Quant à Philippe Lejeune (1986, p. 69), théoricien de l'autobiographie, il ne nie pas son admiration pour l'entreprise littéraire de Doubrovsky : "Les ruses machiavéliques de Doubrovsky m'ont fasciné », assure-t-il, parce qu'elles se situent « dans une zone frontière, à cheval entre deux systèmes de communication : celui de la vie réelle [...] et celui de la littérature de fiction ». En outre, selon Vincent Colonna (2004, p. 13), l'autofiction est une possibilité de lire les œuvres différemment, d'en éclairer certains aspects : «Prise au sérieux, dotée d'une compréhension conséquente et reformulée dans ses principes et ses moyens, cette mythomanie littéraire devient un instrument de lecture prodigieux. Tour à tour macroscope et microscope, elle permet de reconsidérer des phénomènes d'écriture apparemment marginaux, de découvrir des plaisirs littéraires inconnus, des émotions inédites. Une immense perspective s'ouvre alors sur la littérature, quantité d'œuvres et d'écrivains, mal distribués entre l'autobiographie ou le roman, entre la fantaisie et le factuel, apparaissent sous un jour nouveau, révèlent des beautés propres, une logique méconnue. »

Par suite, les débats sont vifs et animés. Les expressions employées pour parler, décrire et critiquer l'autofiction sont nombreuses et vont dans tous les sens. Or, dans cette étude de 
«Mes Mauvaises Pensées », nous allons tenter de percer le mystère de cette forme de littérature intime à travers l'application de dix attributs du concept doubrovskien conçus par Philippe Gasparini.

\section{L'autofiction en pratique}

Philippe Gasparini (2008, p.209) se propose de contribuer à éclairer cette ambiguïté théorique afin de permettre aux lecteurs de séparer le bon grain de l'ivraie, pour ainsi dire. Sans prétendre en établir une liste exhaustive, ni définitive, il en dénombre dix : 1- l'identité onomastique de l'auteur et du héros-narrateur ; 2le sous-titre : "roman" ; 3- le primat du récit; 4- la recherche d'une forme originale ; 5- une écriture visant la "verbalisation immédiate" ; 6- la reconfiguration du temps linéaire (par sélection, intensification, stratification, fragmentation, brouillages...) ; 7- un large emploi du présent de narration ; 8- un engagement à ne relater que des "faits et événements strictement réels" ; 9- la pulsion de "se révéler dans sa vérité", et 10- une stratégie d'emprise du lecteur. Nous allons par conséquent les traiter dans l'ordre.

1- L'identité onomastique de l'auteur et du héros-narrateur

L'autofiction impose une identité assumée par les trois instances narratives, à savoir l'Auteur, le Narrateur et le 
Personnage principal. II s'agit de recourir au prénom, au nom, voire au pseudonyme identifiant directement l'auteur.

Toutefois, Philippe Vilain (2010, pp. 475-476) considère que le "je" autofictionnel peut rester anonyme : «Employant un je qui ne désigne pas nominalement un autre personnage comme dans le roman autobiographique, l'auteur n'a pas besoin de se nommer pour suggérer que l'histoire qu'il raconte le concerne. [...]. On l'aura compris, à travers ce critère anominal, c'est le nœud gordien de ma propre entreprise littéraire que je livre. "

La formule d'identité des instances narratives $(A=N=P)$ est bien appliquée dans «Mes Mauvaises Pensées" à travers l'utilisation du "je" autofictionnel. Ce "je" est le premier mot, le premier pronom omniprésent qui constitue en quelque sorte la charpente de l'œuvre. II est celui de Nina Bouraoui qui dévoile, dans une interview accordée à $\mathrm{F}-\mathrm{O}$. Giesbert dans l'émission littéraire Culture et Dépendance du 08 octobre 2005, que son récit, objet de notre étude, raconte l'histoire de la thérapie hebdomadaire qu'elle a dû suivre chez une psychiatre durant une période de trois ans. Elle dit à ce propos : « Je suis honnête...Je voulais non seulement restituer la parole de ce qui se passe dans le cabinet d'un thérapeute...Parce qu'il y a un lien entre l'écriture 
et l'analyse, on parle de mémoire, de langage et de souvenir » (Giesbert, 2005).

\section{2- Le sous-titre : "roman"}

L'œuvre en question porte une indication paratextuelle qu'est la mention du "roman" sur sa couverture. Ceci exclut tout rapport avec la réalité et place la diégèse au cœur de la fiction. Autrement dit, l'auteure revendique que son but est de romancer/fictionnaliser son histoire personnelle : « Mon travail d'écriture est aussi un travail de faussaire. II n'y a que ma vérité [...], il n'y a que mon interprétation des choses » (MMP, p.206). Elle est encore éprise par le charme du roman et dit : « J'ai toujours vécu dans la magie du roman » (MMP, p.55).

\section{3- Le primat du récit}

L'autofiction doit principalement être un récit en prose narrative. La narratrice autodiégétique s'adresse à un "vous", à un narrataire intradiégétique, au Docteur $C$, une femme à qui elle se confesse et relate ses problèmes psychiques en revenant sur des scènes qui remontent à son enfance, à sa jeunesse, aux rapports qu'elle entretenait avec les membres de sa famille et à sa vie amoureuse. En focalisation interne, la voix de la narratrice se répand en écho visant un « effet de confidence » (Benmahamed, 2000 : p. 45). La narration est intercalée car les 
événements qu'elle essaie de décrire sont filtrés par sa mémoire et donnent ainsi une impression subjective. Il est à noter que le nom de sa famille «Bouraoui » est composé de deux syllabes : "bou" (déformation de abou) signifiant en arabe "père" et "raoui" qui signifie "raconteur", ce qui suggère l'héritage de la culture arabe en l'art de raconter des histoires : Mille et un contes. Le père de Nina préfère les romans de sa fille à son journal intime, « il déteste cette forme, de la vie annotée, répertoriée, [...]; et estime qu'un livre «doit épouser son lecteur et non l'inverse » (MMP, pp.13-14).

\section{4- La recherche d'une forme originale}

L'autofiction est une expérimentation littéraire qui aspire à un style spécifique. Elle s'emploie à enfreindre les conventions traditionnelles de la littérature romanesque pour explorer des horizons fictionnels nouveaux afin de mieux exprimer le moi dans toute sa profondeur. D'ailleurs, l'autofiction « est généralement perçue comme la manifestation emblématique de la nouvelle "ego-littérature" [...] se livrant aux jeux exaspérés d'un certain exhibitionnisme psychologique »(Forest, 2001 : p. 13). Le préfixe "auto" qui signifie "soi-même" en rapport avec un "ego" souverain pourrait être en rapport avec un "autre soi-même", "l'alter-ego". Et dans un cadre fictif, "l'autofiction" se révèle 
indissociable d'une «alter-fiction» (Richard, 2013 : p.11). Expliquons-nous : le texte connote plus ou moins la liberté, l'absence de freins, les pulsions de vie : « Je suis sans limites» (MMP, pp.85-87) comme l'affirme l'auteure-narratrice. La même expression est dite par un autre protagoniste « la Fille à l'héroïne " (cf. MMP, p.85) qui avoue à l'auteure-narratrice qu'elle prend de la drogue et en décrit l'influence sur son état et sur son corps. Elle est, en fait, un personnage évocateur. Ses mots nous font plonger dans les zones interdites de l'inconscient. Les paroles en italique, qui s'étendent sur quatre pages, nous suggèrent que cette Fille est une autre "héroïne", un "moi-délire" qui communique avec le "moi-réel" de la narratrice (cf. MMP, pp.72-76). II nous paraît que l'écrivaine autofictionnaire introduit un personnage supplémentaire dans son récit. Une façon de fixer une identité que Ricœur (1990) a nommée " l'identité narrative » dans «Soi-même comme un autre ». Bouraoui nous dit à propos de ce personnage qu'elle ne l'aime pas mais qu'elle s'attache à elle pour la simple raison qu'elle est tout à fait son opposé (cf. MMP, p.96). Ce qui fait que "soi-même" est ici employé dans le cadre d'une comparaison ; il est "autre", "contraire", "distinct". Quoique l'auteure-narratrice ait besoin de la voix de "la Fille à I'héroïne", de ses mots et son langage, elle s'est libérée d'elle et de sa folie (cf. MMP, p.98). Régine Robin (1997, p. 24) disait 
justement que «l'autofiction c'est, en quelque sorte, l'identité narrative se reconfigurant, mais se défaisant en même temps qu'elle se tisse. "C'est pourquoi, l'autofiction lui sert à se (re)construire une/des identité(s) à l'abri des regards indiscrets.

\section{5- L’écriture visant la "verbalisation immédiate"}

L'autofiction entend transcrire, par le biais du langage, les souvenirs de l'auteur tels qu'ils lui viennent à l'esprit. Dans Mes Mauvaises Pensées, les phrases sont brèves, saccadées, en raison du recours massif à l'itératif. Nous trouvons des mots qui mettent l'accent sur une mémoire chaotique, non maîtrisée telle une obsession comme «peur» (cf. MMP, pp. 9, 10, 20, 76, 94, $216, \ldots$ etc.), « mort » (cf. MMP, pp.17, 22, 24, 27, 36, ...etc.), « nuit » (mot répété 12 fois dans la même page, MMP, p.133) « vide» (mot répété 8 fois dans la même page, MMP, p.137). II y a aussi des phrases comme : «Qui suis-je » (cf. MMP, pp.58, 61,135-136), " je suis coupable » (cf. MMP, pp.99-100), « je me détruis de l'intérieur » (cf. MMP, p.171). Une écriture du trauma, qui se traduit dans la répétition en tant que principe constitutif des mauvaises pensées de l'écrivaine. Celles-ci constituent le noyau du récit et comportent une interprétation traumatique, conséquence du sentiment de déchirure existentielle et de déracinement éprouvé par l'auteure-narratrice. 
De ce fait, l'autofiction, qui lâche la bride au langage vers une écriture spontanée, serait ainsi « l'autobiographie de l'inconscient » (Jenny, 2003). C'est une écriture de cure où se donne à nue une profondeur d'un Moi en émoi. Le passage suivant en témoigne :

«Pour effacer mes mauvaises pensées, je tiens un carnet; elles sont numérotées, je pourrais vous les lire, sortir le carnet de mon sac, mais j'ai encore honte, lire ce que j'écris est d'une grande intimité, surtout ici, dans votre cabinet qui pourrait être une chambre ; de mémoire, je peux vous dresser ma liste de phobies, 1. L'cil de ma voisine qui mange son visage; 2 . Me jeter par la fenêtre ; 3. Descendre au fond de moi, comme un objet détaché de ma conscience; 4. Au cinéma, le rang devant moi, les gens n'ont plus de cheveux ; 5. Mordre au visage ; 6. Partir sans payer ; 7. Ne plus maîtriser mon langage ; 8. Grogner ; 9. Blesser un enfant ; 10. Avoir une image fixe dans la tête : une petite planche de bois, trouée sur l'une de ses extrémités » (MMP, pp.82-83).

II s'avère que l'auteure-narratrice est consciente de son inconscient. Elle autorise l'accès de ses idées inconscientes à l'espace conscient à travers « l'écriture (qui) sait des choses que l'écrivain ne sait pas» (Vilain, 2005 : p. 221). 
6- La reconfiguration du temps linéaire (par sélection, intensification, stratification, fragmentation, brouillage...)

L'autofiction ne prétend pas retracer une vie personnelle selon une logique chronologique. Elle propose au contraire une démarche consistant à réfléchir sur la transcription des évènements tels que la mémoire les fabrique (une mémoire qui peut être déficiente). C'est dans ce sens que Burgelin (2010, p.216) indique que, dans les textes d'écriture de soi, « la mémoire est la grande ordonnatrice du récit et de mise en scène.»

C'est ainsi que, comme le souligne Laflamme, (2006, p. 48), Bouraoui se plonge « dans l'anarchie de la mémoire ». Les souvenirs sont cités avec une mémoire désagrégée et trouée. L'auteure se reproduit inlassablement à travers une « mémoire inquiète» (Viart, 1997) de sorte que son souhait est d'inventorier des événements, des souvenirs et des émotions disparates afin de les préserver de l'oubli : « Je rêve d'un livre de transformation, qui m'aurait suivie depuis mon enfance, je rêve d'un album, je rêve d'un almanach ; je dois tout écrire pour tout retenir, c'est ma théorie de l'écriture qui saigne » (MMP, p.21).

Pourtant, elle doit arrêter cette hémorragie d'écriture et bloquer le flux des souvenirs. Elle procède, après avoir traqué les vestiges de son histoire traumatique, à une opération esthétique 
; elle esthétise sa mémoire en se détournant de "l'avant" au profit de "l'après" comme l'illustre ce schéma :
L'avant
le pendant et l'après

Une écriture saignante

une esthétisation de la mémoire

Elle déclare : « Je dois avancer, je dois exister » (MMP, p.111) car il ne faut pas se figer dans le passé mais suivre la route du temps.

\section{7- Le large emploi du présent de narration}

L'autofiction est l'écriture de l'instant présent, même si les souvenirs transportent l'auteur dans le passé. Ce qui est frappant dans Mes Mauvaises Pensées c'est la force de la réminiscence qui ramène l'Algérie en France de sorte que le présent français porte l'empreinte du passé algérien. En d'autres termes, le passé est toujours présent : le retour continuel au passé, à la rétrospection est dû à la rupture brutale que Nina a endurée lorsqu'elle a appris lors, de ses vacances en France, qu'elle ne rentrerait pas en Algérie, laissant ainsi derrière elle la ville de son enfance, sa chambre, ses souvenirs, bref sa "première vie " (MMP, p. 101). C'est la « nostalgérie » (Robin, 2002 : p.208) qu'éprouve l'auteure-narratrice envers son pays et son origine algérienne : "Il y a ce mouchoir blanc que je n'ai pas agité " 
(MMP, p.267). Elle cherche alors à supprimer la distance entre le temps vécu et le temps narré, en employant le présent de narration, ce qui devrait permettre « de rendre la chose immédiate aussi bien pour l'auteur que pour le lecteur "(Vilain, 2005 : p.185). Cela dit que voir les actes de mémoire se matérialiser devant soi est bien ce que Maurice Blanchot (1959, p. 31) entend par « la transmutation du souvenir en une réalité directement sentie ». Voilà pourquoi, bon nombre de ses organes sensoriels sont des supports de réminiscence visant « la recréation d'émotions immédiates » (Doubrovsky \& Contat, 2001 : p. 120). Tout son corps est parfaitement capable de mettre en forme ses souvenirs : "Il y a tant de sensualité, sur ce rocher plate-forme, dans la terre rouge, sous l'eau chaude et profonde, dans l'odeur aussi, que je retrouve sur le petit chemin du Cap-Martin » (MMP, p.200).

8- L'engagement à ne relater que des « faits et événements strictement réels "

L'autofiction se veut essentiellement référentielle dans le sens où elle relate des faits et évènements réellement vécus par l'auteur. L'étiquette "roman" indiquée sur la couverture devrait nous prémunir « en principe contre toute lecture référentielle » (Colonna, 1989 : p.174), donnant un effet de fiction. Cependant, 
les allusions et les références personnelles qui y figurent confirment le caractère véridique de sa vie éclairée par des éléments biographiques et des entretiens, telle que sa date et son lieu de naissance : "le 67. C'est ma date de naissance, c'est l'année où je suis arrivée à Alger » (MMP, p.44), sa personnalité en tant qu'auteure : « Je redeviens l'auteur qui passe à la télévision, qu'on équipe d'un micro, qu'on maquille, qu'on questionne» (MMP, p.51). Elle nous confie aussi : "Souvent, je préfère lire au lieu d'écrire, parce que la lecture m'arrache au réel, tandis que l'écriture - mon écriture - m'oblige à m'y tenir au plus près » (MMP, p.51).

II est à indiquer qu'hormis les noms de personnes célèbres cités dans le récit comme Hervé Guibert, Eileen Gray, Le Corbusier, David Lynch, nous constatons l'utilisation d'un code onomastique très restreint. Bouraoui emploie les noms communs pour désigner sa famille, (mon père, ma mère, ma sœur, ma grand-mère, mon grand-père, etc.), ses liens amoureux : Diane de Zurich, la Chanteuse, l'Amie, les trois garçons de Biarritz, Johan. Elle ne nomme pas ses personnages principaux/secondaires mais les mentionne souvent par des lettres alphabétiques : M., le docteur C., Madame B, Monsieur S., etc. Ce qui explique le souci de confidentialité de l'auteure- 
narratrice dans le but d'éviter toute accusation d'atteinte à la vie privée qui pourrait être soulevée par ces personnes réelles.

\section{9- La pulsion de "se révéler dans sa vérité"}

L'autofiction s'inscrit dans une démarche analytique/psychanalytique où l'auteur est dans une quête de soi et de la vérité sur soi. Nous proposons donc une mise en lumière de l'écriture de l'auteure-narratrice, qui est en fait un moyen d'affirmer son existence, de même que de ses relations avec les différentes personnes qui ont joué un rôle important dans sa vie comme sa famille et ses amours, ainsi que des espaces identitaires qu'elle a fréquentés. Ces éléments contribuent à fouiller dans le tréfonds de l'âme de Bouraoui à la recherche d'elle-même.

Nous pouvons diagnostiquer un véritable malaise existentiel qu'elle tente de surmonter via l'écriture littéraire. « L'écriture m'a transformée, je crois, je ne suis plus la même » (MMP, p.152). Elle avoue que la disparition de l'écriture est semblable à celle de toute son existence. L'extériorisation de ses tourments existentiels ne sera réalisée que par l'écriture : «Les livres sont comme des bras, je me couche dans cette chaleur-là » (MMP, p.47). L'écriture apparaît en conséquence comme un instrument thérapeutique, une cure psychanalytique. 
Serge Doubrovsky (1993, p. 213), père de l'autofiction, constate qu'un auteur qui parle de son vécu « se dépiaute » et «taille dans ses chairs » C'est exactement ce que fait Bouraoui de son moi qui est en crise et en proie à des humeurs inconstantes. Elle formule ainsi son mal-être : « Il y a le bruit de ma honte, qui descend au fond de moi, et c'est encore ce bruit quand je dois défendre un livre, quand je dois m'expliquer, quand je dois dire qui je suis vraiment. Il y a des échappées en moi, il $y$ a des parties invisibles » (MMP, pp.44-45). Et pour nous faire part de ce qu'elle ressent, l'auteure-narratrice use d'un vocabulaire aussi fort que le sentiment qu'elle éprouve :«C'est la solitude qui tue, c'est elle qui ronge les sangs, rongée, c'est cela ma vie, elle est rongée » (MMP, p.89).

Mais comment remédier à ce «moi, en tant qu'objet perdu» (MMP, p.266) ? En réfléchissant à un moyen de s'en sortir, une seule action lui paraissait salvatrice : retourner nécessairement au moment où tout a basculé dans sa vie : «Je suis ramenée à mon point de fuite, je suis ramenée à ma ligne algérienne qui est la ligne de départ d'une course de fond : ma vie» (MMP, p.267). Et c'est à ce moment-là qu'elle avoue n'avoir jamais cessé d'épier cette vérité profonde. Raison pour laquelle elle a toujours eu le regard tourné vers le passé : « Je n’ai rien fermé parce que 
je ne veux pas fermer, je ne veux pas qu'une partie de moi se meure » (MMP, p.223). Maintenant qu'elle est dedans, elle pense y rester le temps de démêler le nœud dont elle est l'objet. Elle tente d' " ouvrir les portes de [so]n enfance et chercher ce qui manque ou ce qui traverse » (MMP, p.27).

Un autre malheur à notifier provient de sa « peau buvard» (MMP, p. 66), peau qui s'imprègne de tout et des autres, c'està-dire de l'histoire de l'auteure-narratrice, et de celle des membres de sa famille. «Ce que je suis tient peut-être dans le coeur de cette famille, je dois encore les regarder, pour me retrouver» (MMP, p.84). Elle devient un « sujet buvard» (MMP, p.28) qui a pour tâche de relayer ces histoires, d'en porter la mémoire.

$\mathrm{Au}$ fait, être de mère française et de père algérien, c'est "venir de deux familles que tout oppose, les Français et les Algériens » (MMP, p.53) ; l'emploi du mot "famille" pour désigner les Français et les Algériens montre bien l'inséparabilité, chez Bouraoui, de l'histoire personnelle et de l'histoire collective.

II s'agit toujours de cet amour envahissant, de ce lien fusionnel avec ses parents ; elle déclare : " Je suis faite de ce ciment» (MMP, p.18). Elle leur témoigne sa reconnaissance car 
ils l'ont énormément soutenue et encouragée dans son travail d'écrivain.

Commençons par sa relation très particulière avec sa mère qui est son ange gardien, son pilier et son bouclier : "Ma mère est ma forteresse, ma mère est ma boule d'amour " (MMP, p.125). Elle est aussi son idole; la répétition du procédé comparatif « comme elle» le prouve (cf. MMP, p.194). Nina offre à sa mère un rang privilégié au sens premier du terme en la parant des attributs de l'astre solaire : "Ma mère a été choisie par le soleil » (MMP, p.125). Celle-ci garde donc une place dominante auprès de sa fille : «Ma mère occupe tous mes territoires " (MMP, p.254). Rien d'étonnant en cette relation fusionnelle que ressent tout enfant à l'égard de sa mère. Mais à l'adolescence, la volonté de se fondre avec sa mère va jusqu'à penser à la faire entrer dans le cercle du désir charnel : « Je suis au-dessus de la mer, comme je pourrais être au-dessus de ma mère " (MMP, p.32). L'emprise affective de la mère rend Nina toujours en proie au sentiment de culpabilité de l'avoir quittée. Elle voulait être la substitution symbolique du père pour compenser l'absence de son départ.

Pour elle, occuper la place de son père c'est aussi rendre possible et légitime le désir qu'elle éprouve à l'égard de sa mère: 
«Quand je m'installe face à ma mère, je deviens la doublure de mon père » (MMP, p.254). Nina se perçoit par ailleurs comme la protectrice de sa mère : « Je suis le chevalier de ma mère, je réponds à ses appels au secours, je fais passer son corps avant le mien, j'ai la mémoire de ses maladies, j'ai souvent voulu être malade à sa place» (MMP, p.30). Elle a cependant peur de cette union à cause de sa liaison si forte avec sa mère : «Ma peur est la peur du lien avec ma mère, ma peur est la peur de cet amour, ma peur est la peur de ma mère qui ne sait pas séparer son corps de mon corps» (MMP, p.102). En bonne lectrice de Freud, elle nous explique les méfaits de cette symbiose : «Dans L'Homme aux loups de Freud, il se passe quelque chose avec l'enfant quand il lance sa balle et qu'elle ne revient pas ; [...] ; quand ma mère disparaît derrière le champ, les marguerites deviennent mes loups : pour moi, elle ne reviendra pas » (MMP, p. 43). Nous savons que "l'Homme aux loups" est l'histoire d'une névrose infantile (cf. Mijolla, 2005 : p.1161). Dans ce rêve effrayant, Serguei Pankejeff s'identifie à la mère et l'angoisse éprouvée envers le père est déplacée sous la forme de la phobie du loup. Quant à Nina, elle sent que sa mère est en perpétuel danger quand elle se sépare d'elle. Alors tous les éléments qui font obstacle à leur attachement l'une à l'autre deviennent des 
loups, des animaux féroces et sanguinaires, présentés comme des ennemis de l'Homme.

Néanmoins, si la coupure du cordon ombilical amène une distanciation physique nette du bébé par rapport à sa mère, le sentiment d'être séparée d'elle et de constituer ainsi une entité individualisée n'apparaîtrait qu'avec l'émergence pulsionnelle que commence à ressentir la pubertaire. Nina, comme tout adolescent, désire s'investir dans cette nouvelle phase et vivre sa "libido" loin de la figure maternelle : « Je suis gênée d'être si près du corps parce que je ne suis plus une enfant " (MMP, p.103). La mère semble avoir perdu le contrôle sur son enfant qui ne conçoit sa puberté que là où elle n'y est pas : « Ma mère me téléphone tous les soirs et parfois je ne veux pas lui parler, parce que je me détache de son corps, de cet amour inouï qui me donne le vertige» (MMP, p.56). La mise à distance est donc justifiée par la crainte de retomber en enfance : "Sa voix me remet dans les jours de l'enfance» (MMP, p.56).

Donc, "se désidentifier" de sa mère est le mot d'ordre qu'elle scande pour briser les amarres qui l'attachent à elle. Une petite voix à valeur thérapeutique surgit alors d'elle pour lui expliquer qu'elle doit se dégager du corps et du mental de sa mère : «Je ne peux pas réparer l'enfance de ma mère par ma propre enfance 
»(MMP, p.115). Elle doit s'arrêter d'être un autre "je(u)" de sa mère.

Quant à son père, elle le voit comme un exemple dont elle désire être le reflet. Elle l'aime et l'estime profondément. Si l'auteure-narratrice s'identifie à son père, c'est notamment parce qu'elle convoite la position de «chef de famille » (MMP, p.123): « Je suis le fils de mon père, je suis surtout son miroir, un jour il dit : "Tu es le jeune homme que j'étais" (MMP, p.185). Elle sent que son père la considère comme son fils. Il lui a inculqué les comportements masculins et l'a même surnommée « mon brio » (MMP, p.184).

En outre, le processus d'identification totale à son père va jusqu'au bout ; elle explique son désir pour les femmes parce qu'elle le prend comme modèle : «C'est si simple de comprendre aujourd'hui mes amours, je marche dans les pas de mon père, et je sais comme lui combien il est enivrant de suivre le parfum d'une femme, de répondre à sa voix, de soutenir son regard » (MMP, p.186).

II joue aussi le rôle de guide pour sa fille dans sa quête de soi ; il lui montre que se questionner sur soi, c'est se donner la possibilité de se reconnaître soi-même, de reconnaître l'autre en soi, et d'être reconnu par l'autre, dans le but de s'identifier et de 
se redéfinir. L'auteure-narratrice nous fait part des propos de son père lorsqu'elle est en Suisse : «I/ dit que ce que je suis en train de vivre - le dépaysement - est fondateur pour mon avenir, qu'il faut visiter le monde pour se connaître soi, ce que je comprends ainsi : il faut visiter les autres pour se savoir soi» (MMP, p.50).

Après avoir mis l'accent sur le rôle de ses parents, nous allons focaliser l'attention sur sa relation avec ses grandsparents et plus précisément ses grands-pères : "Mon grandpère algérien est dans ma mémoire, mon grand-père français est dans le silence » (MMP, p.104).

Elle décrit son grand-père paternel ainsi : « // est blond avec des yeux clairs sur les photographies, il a le visage très fin " (MMP, p.45), mais elle sait très peu sur lui et tente de retrouver un peu de lui sur son corps à elle : «Quelle était son enfance? Je ne sais pas. Qui étaient ses parents? Je ne sais pas. I/ avait une moto, il jouait de la mandoline, il achetait des champs d'cillets à Nice, il avait un don pour le langage et la conversation. Il y a sa peau sous la mienne, il y a son nom, il y a ses yeux, il $y$ a ses mains sous mes mains " (MMP, p.45). Nous voyons comment elle creuse pour chercher les racines profondes de ses origines paternelles afin de se constituer une image et une identité algérienne. 
Pour le grand-père maternel, français, au parfum « Chanel Monsieur », "dont le visage reste absent de (s)es souvenirs » (MMP, p.22), il occupe une place importante dans le récit. L'auteure-narratrice explique qu'il a toujours été froid avec sa mère, qu'il ne lui a jamais montré d'amour et qu'il n'a jamais, non plus, accepté son mariage avec un Algérien. Le grand-père, chirurgien à Rennes, assène à sa fille quand elle se marie : «Tu finiras mal !» (MMP, pp.84, 90, 100, 240). Une phrase qui se répète comme un leitmotiv que la petite-fille Nina essaie d'éviter mais elle n'y arrive pas, elle aussi pense que ça devrait « mal finir» (MMP, p.197) comme le croyait son grand-père.

Elle le décrit quand la colère le prend : « // ne se retient plus, il parle, il parle, il parle de l'Afrique, de l'Irak, du Maghreb, il a peur de tout, il a peur qu'on lui vole son argent, ce que j'entends ainsi : on lui a déjà volé sa fille» (MMP, p.162).

Sa mère aussi se met en colère quand le grand-père dit qu'il a acheté son journal de chez le b. Elle se lève et crie contre lui : «Pas devant mes filles. Tu peux tout me faire, mais pas devant mes filles» (MMP, p.19). Ici, l'auteure-narratrice s'est contentée de mentionner la lettre $b$ pour ne pas heurter le lecteur surtout s'il est d'origine maghrébine. Nous devinons que "b" désignerait 
"barbare" employé de façon péjorative de la part du grand-père maternel.

En effet, sa relation avec la mère de Nina est basée sur la peur : « Je n'ai jamais pu regarder mon père dans les yeux tant j'ai peur de lui » (MMP, p.144). Pour l'écrivaine, son grand-père est la cause principale des crises d'asthme de sa mère. Elle affirme, par exemple, que sa mère est «prisonnière de son père qui l'étouffe» (MMP, p. 144). Elle a donc de mauvaises pensées à son égard : " Je pense qu'il faudrait que je tue le père de ma mère pour qu'elle respire enfin » (MMP, p. 87).

Continuons l'évocation des liens et en particulier les relations amoureuses que l'auteure-narratrice tisse dans son roman et que nous allons exposer successivement, à savoir le docteur $\mathrm{C}$, Madame B., Diane de Zurich, la Chanteuse, l'Amie ainsi que ses relations avec $A$., le garçon de La Baule, les trois garçons de Biarritz, et Johan.

De prime abord, le face-à-face avec une psychiatre, le huis clos avec elle dans son cabinet où tout le petit monde de Nina est remonté de ses profondeurs, a fait naître un amour pour le docteur C. : « Je tombe amoureuse quand je pense à vous » (MMP, p.271). Nous n'entendons pas la voix du docteur C. tout 
au long du récit mais elle a un rôle si important qu'elle est une des dédicataires du livre.

Le personnage de Madame B., amie de sa mère, constitue effectivement la première femme pour qui l'auteure-narratrice éprouve du désir. «Première strate romantique » et véritable « coup de foudre », elle s'avère une « idole » (MMP, p.198) pour elle.

Durant I'hiver 1980, la mère de Nina manque de mourir d'un œdème. Afin qu'elle puisse se reposer, des amis de la famille prennent soin de ses enfants. L'écrivaine se retrouve alors chez Madame B. dans l'appartement de cette dernière, parfaitement consciente de son corps gorgé de désir. Elle conçoit l'effet qu'elle peut produire sur Madame $B$. et entre dans une dynamique de séduction : « Je crois que j'aime vraiment Madame B., je sais la regarder, l'envelopper, l'écouter, je sais aussi l'effet de ma tendresse sur elle, je sais ce qui la fait fondre, je sais aussi qu'elle s'attache, encore plus vite que moi, encore plus fort que moi » (MMP, p.66). Mais en dépit de la force du désir qu'elle éprouve pour Madame B., elle sait très bien que ce qu'elle ressent envers l'amie de sa mère n'est pas réciproque : «Ce désir a un lien avec la mort, parce que c'est un désir non partagé. Madame $B$. a de la tendresse pour moi, c'est tout. [...], elle retire vite mes 
bras de ses épaules, quand je l'embrasse avant de dormir. Elle sait qui je suis » (MMP, p.135).

Vient ensuite la vie à Zurich avec Diane de Zurich, " c'est l'effet d'un désir boomerang» (MMP, p.35). Chronologiquement, la première de ces relations amoureuses est celle que l'auteurenarratrice partage avec Diane. II s'agit d'une relation inégalitaire marquée par la tristesse, l'absence et le manque, vécue sous le signe de la dévalorisation et de la haine où notre écrivaine, dépendante du regard de Diane, a l'impression de ne pas pouvoir entrer dans la vie de celle pour qui elle éprouve une puissante attirance et un attachement intense : "Je n'entre pas dans son cadre, je n'entre pas dans sa vie, ma main ne tient pas dans la sienne, nous sommes si différentes que nous nous dévorons" (MMP, p.175). Cette relation est destructrice et aliénante : «Avec Diane de Zurich, l'amour a un lien avec la mort» (MMP, p.174).

Après avoir été amoureuse de Diane, l'auteure-narratrice fait la connaissance de celle qu'elle nomme "la Chanteuse », qui « est née à Alger» (MMP, p. 219) et qui correspond en fait à la chanteuse de sa jeunesse. Le couple qu'elles forment consiste en une relation amour-haine qui se fonde d'abord et avant tout sur la peur et l'effacement de soi de la part de l'auteurenarratrice : «Je suis restée avec la Chanteuse parce que j'avais 
peur de la solitude et que j'avais peur de moi » (MMP, p.161). À un moment de sa vie, l'écrivaine est dépossédée par la Chanteuse : «Le succès des autres est une prison ; je vis dans sa voix, dans ses tournées, dans ses vacances, j'ai encore besoin d'un corps, je veux qu'on me serre dans les bras, c'est toujours moi qui essuie ses larmes, c'est toujours moi qui recueille l'histoire de son enfance ; avec elle, je suis sans passé, [...] avec elle, je perds mon nom » (MMP, p.34).

D'ailleurs, elle arrête d'écrire durant les deux années qu'elle a vécues en couple avec la Chanteuse ; une relation égoïste qui ne tient pas compte des besoins de son partenaire : « Je m'efface pour elle, je m'efface de moi, il y a ces mots un jour au sujet de la disparition de mon écriture : "Cela me plaît que tu n'écrives plus, il n'y a pas (de) place pour deux" " (MMP, p.172).

Seule la relation que l'auteure-narratrice développe avec l'Amie est axée sur un regard d'attention ou d'intérêt mutuel. L'Amie de Mes Mauvaises Pensées est à la fois personnage et dédicataire du livre : «L'Amie est à la source de mes livres » (MMP, p.30). Elle est perçue comme le double de la narratrice en raison de leur ressemblance frappante : « J'ai l'intuition d'une autre vie ou d'une double vie, puisque je suis le reflet de l'Amie et que l'Amie est mon reflet, puisque nous nous amusons de 
cela, de notre lien - miroir» (MMP, p.105). La confusion entre les deux atteint parfois son paroxysme de telle sorte que l'on peut croire que ce nom commun est peut-être le pseudonyme par lequel la romancière se désigne : « I/ y a un prolongement des sangs, une adoption de l'une par l'autre " (MMP, p.35). L'interchangeabilité des rôles et des identités entre les deux essences montre que l'Amie n'est que l'âme sœur dans un autre corps de Nina Bouraoui. Cette intimité est formulée dans ce passage : «Quand je regarde ma mère sur le balcon de la Résidence, l'Amie regarde sa mère au troisième étage de la rue Pierre-Nicole, quand je danse au Grenel's, I'Amie danse à l'Apoplexie, quand je veux faire du cinéma, l'Amie passe un casting avec A.S, [...]» (MMP, p.256). Encore dit-elle : « J'ai souvent pensé que je venais de l'Amie, que nous avions une circulation de nos deux existences, de l'une vers l'autre » (MMP, p.263).

La relation entre elles se double : c'est que chacune des deux s'est fortement identifiée à son père. L'Amie a perdu le sien jeune, mais elle est restée très proche de lui. II revêt une importance capitale dans sa vie, comme c'est aussi le cas pour l'auteure-narratrice dont le père est cependant toujours en vie. 
Toutes deux ont l'impression de suivre les traces de leur père; elles croient qu'elles sont le prolongement de leur progéniteur et toutes deux se révèlent soucieuses de la fierté que leur père peut ressentir à leur égard. À ce sujet, l'Amie dit à son amoureuse : «Tous les jours, je me dis qu'il serait fier de moi. Je suis sa ligne de vie tu sais, d'une certaine façon, je poursuis son ouvrage »(MMP, pp.259-260). L'identification de Nina à son père est totale : «J'ai ses mains et sa peau, j'ai cette chair fossile qui nous lie. J'ai voulu écrire pour répondre à ses cartes postales, j'ai voulu écrire pour qu'il soit fier de moi » (MMP, p.15).

À ce propos, il nous convient de mettre en évidence la relation de l'auteure-narratrice avec les hommes. II s'agit d'une relation d' « image-miroir » (MMP, p.38). Bouraoui exalte la force physique des hommes. À La Baule, en raison de la présence d'A., le professeur de plongée qui la fascine, elle a le projet de créer un livre : " J'ai un livre entier dans ma tête, ce serait un livre sur les hommes, sur A., [...], sur son ventre dur et ses épaules fortes, sur son sourire, sur sa peau, sur sa main qui prend la mienne sous l'eau, [...]» (MMP, p.117). Ce livre serait aussi, dit-elle, écrit sur la beauté de trois jeunes hommes que l'auteure-narratrice admire un jour sur une plage de Biarritz : 
«Ce livre serait aussi sur les trois garçons de Biarritz, [...], sur les corps tendus, gavés de plaisirs, les corps de ces trois garçons si beaux, [...], je les surveille, chaque jour, fascinée, essayant d'entrer dans cette boule de feu, dans leurs jeux, essayant de les décrire avec des mots, de restituer la douceur, et la violence, de leurs gestes, de leurs courses, de leur désir qui semble envahir la plage, brûler les sables et les rochers » (MMP, pp.118119).

L'auteure-narratrice, à l'époque où elle vit encore en Algérie, s'identifie donc aux hommes, mais se perçoit alors sous l'angle du manque, comme le souligne d'ailleurs le titre d'un de ses livres, Garçon manqué (2000), publié avant Mes Mauvaises Pensées.

L'ambivalence de genre est incarnée ainsi par le personnage de Johan. Il s'agit d'un de ses camarades de classe en Algérie. II ne parle pas, passe beaucoup de temps à la salle de sport et fait de la boxe mais elle croit comprendre la source de son mutisme. Elle est émerveillée par cet être parce que, comme elle, son identité de genre féminin ne correspond pas à son corps d'apparence masculine.

Pendant les vacances où tous vont à la plage, l'auteurenarratrice, captivée par Johan, qui reste habillé supposément en 
raison de la fragilité de sa peau, décide de se rapprocher de lui: « Il y a mon corps qui se détache du monde, parce que je sais, je sais mon désir pour Johan, je sais cette part de moi, si profonde et si légère à la fois, je sais cette vie heureuse, je sais. Je sais que Johan est une fille » (MMP, p.205). Ce mélange d'identité sexuelle fascine l'écrivaine qui reverra Johan par hasard en Allemagne vivant pleinement sa nouvelle identité : «Je sais aussi que je le retrouve, ou je la retrouve, bien des années plus tard dans une université de Berlin ; c'est un bal de filles, je descends l'escalier, je le reconnais vite, à cause du visage qui n'a pas changé, à cause des yeux et du regard froid, il est ou elle est avec une autre fille, ils dansent ensemble sur une valse et je le trouve d'une grande beauté parce que je sais, et qu'il ressemble encore à un garçon, et je suis troublée par cela, par ce qui revient sur moi [...]»(MMP, p.205).

Venons enfin dans ce point à la représentation et au rôle des espaces comme Alger/Algérie, Rennes en France, Zurich en suisse et Provincetown aux États-Unis dans l'enquête identitaire de l'auteure-narratrice et la formation de son moi intime.

Nina Bouraoui évoque son rapport fusionnel avec l'Algérie, cet autre double, cet autre soi, cet autre amour. Elle dit avec fierté : « Je suis le coeur de l'Algérie » (MMP, p.25). Cet espace 
autobiographique tient une place prépondérante dans l'œuvre de la romancière. Cependant, le sentiment terrible de se manquer à soi-même disparaît comme par magie dès qu'elle se remémore les instants de bonheur vécu durant son enfance algérienne : «Moi je ne m'intéresse qu'à ma vie, ma vie d'Alger, le parfum des fleurs, la lumière sur le sable, mon corps qui court vers la mer» (MMP, p.52).

L'Algérie est décrite comme un espace sans limites infiniment ouvert et dépouillé de toute forme de barrières : « L'Algérie est ma maison à l'air libre ; ma maison sans toit » (MMP, pp.222$223)$; elle va même jusqu'à l'assimiler à la voûte céleste « par ce ciel bleu et algérien qui semble former (s)on toit » (MMP, p.157).

Pour vanter d'emblée sa patrie, elle use d'un vocable qui suscite les charmes d'une Algérie en accord avec le ravissement: « I/ y a un mystère algérien » (MMP, p.219). Son désir intense de recréer l'ambiance de cette époque lointaine fait surgir dans la sphère du réel quelque chose de l'ordre illusoire.

Cet espace est le lieu qui lui permet de reconquérir son origine culturelle ainsi que tout ce qui est en corrélation avec le bonheur qu'incarne cet "au-delà" magique. L'écrivaine retrouve toute sa féerie : « J'ai des rêves orientaux ; pour moi la magie, 
c'est ma sœur qui chante Fairouz, c'est mon père qui danse sur Abdelwahab» (MMP, p.18).

Ce monde de merveille s'achève à son départ définitif pour la France. La sortie du territoire algérien coïncide avec la phase de sevrage de l'enfance et commence une autre phase de sa vie où se cristallise un autre "moi".

En France, elle renaît dans de meilleures conditions ; elle y trouve le bonheur de vivre librement. Tout lui semble en parfaite harmonie avec ses attentes et ses aspirations : «Je dois l'admettre, je m'y sens bien, peut-être encore plus à l'aise qu'à Alger, parce qu'elle est plus neutre » (MMP, p.111).

Or, cet enthousiasme de vivre autrement se fond à présent comme un glaçon sous le soleil « dans ce lieu si froid (la maison de Rennes), dans ces gens si secrets, dans ces étages si sombres» (MMP, p.158). La raison est clairement explicitée dans ce passage : " Je pense que Rennes est une ville maudite ; je pense que je viens aussi d'ici, de la mort. Alger serait du côté de la vie » (MMP, pp.76-77). Cet avis négatif sur la ville française insinue une crainte implicite d'une intégration réussie qui signifierait en effet l'oubli de ses racines algériennes. Alors, pour remédier à ce sentiment de malaise, elle décide de résumer l'Hexagone en une toile de peinture qui sert à mettre en vedette 
les couleurs de son jardin d'Eden : « Je traverse le parc Monceau, l'herbe, les arbres, les manèges, les balançoires, les cris des enfants, ce décor-là me relie à l'Algérie » (MMP, p.222). Mais il n'y a là rien d'étonnant car ses réminiscences sont une lutte contre l'oubli : «Souvent, je me dis que je fais tout pour reconstruire mon édifice sensuel, j'ai rapporté l'Algérie en France, j'ai rapporté sa douceur et sa violence, et je suis devenue sa douceur et sa violence » (MMP, p.200).

C'est le même cas quand elle part avec sa famille en Suisse à Zurich, elle amène l'Algérie partout où elle va... Et pour revivre ce qu'elle a déjà vécu, elle se laisse projeter dans l'autrefois de sa vie antérieure où règne un printemps éternel. À Zurich, elle ressent le sentiment de n'avoir jamais quitté I'Algérie. Ici : « Je suis dans ma chambre d'Alger, ma chambre est la plus lumineuse de l'appartement, le soleil mange le bois de mes volets, je suis sur mon lit, je transpire, j'ai conscience de mon corps, [...], cette façon de me voir revient dans la chambre de Diane de Zurich » (MMP, p.35).

II est à signaler que l'auteure-narratrice a évoqué le lac de Zurich, « le lac de [s]a jeunesse, le lac des désirs perdus » (MMP, p.40). Les lacs sont ainsi considérés « comme des palais souterrains, de diamant, de bijoux, de cristal, [...], mais qui attirent 
aussi les humains dans la mort. Ils prennent alors la signification redoutable de paradis illusoires » (Chevalier \& Gheerbrant, 1986 : p. 556). II paraît que le lac de Zurich suggère une place artificielle qui ne lui donne qu'une jouissance immédiate et qui échoue à lui faire oublier son Algérie, son paradis perdu.

II y a aussi la forêt du Dolder où elle court pour échapper à elle-même (cf. MMP, p.213). Ce massif boisé zurichois est tout à fait différent de la forêt d'eucalyptus située en Algérie qui lui révèle sa propre vérité dans sa quête d'identité. Elle qualifie cet espace forestier algérien de «terre rouge » (MMP, p.204), allusion faite probablement à la terre de laquelle Dieu a créé l'homme. II nous semble que l'auteure-narratrice cherche à entrer en communion avec la nature forestière car « tout homme y peut redécouvrir sa vraie nature »(Pons, 1999 : p.32). Elle confirme son adhésion complète à ce milieu boisé qu'elle considère comme le lieu de rencontre avec soi.

II n'en demeure qu'un espace très cher à l'écrivaine qu'est Provincetown. Dans cette ville américaine où elle ne connaît nullement les personnes qui l'entourent, elle sent le « fil invisible» (MMP, p.157) qui traverse les femmes, le lien qui les unit.

En ce sens, la ville de Provincetown constitue clairement, pour elle, un espace de liberté. Les affirmations ci-dessous sont 
l'expression de joie d'un esprit libéré de tout ce qui faisait obstacle à son épanouissement :

- « Je suis si bien, si près de moi, dans cette petite ville, [...], je ne me suis jamais sentie aussi solidaire de moi-même " (MMP, p.188);

- « Je ne confonds rien à Provincetown, je sais qui je suis, je sais ce que je désire» (MMP, p.190);

- « Je sais que je reviendrai à Provincetown, c'est mon paradis, [...], je ne me suis jamais sentie bien en moi-même puisque je suis pleine de moi "(MMP, p.195). L'expérience que fait Bouraoui à Provincetown est celle de l'adéquation avec soimême. Dans cette ville de femmes, elle peut être authentique et pleinement autonome. La communauté de femmes a ici un rôle de catalyseur. Elle facilite et favorise les retrouvailles avec soimême et avec "l'autre-en-soi nié, renié et expulsé, mais qui toujours revient jusqu'à ce qu'on l'accueille » (Caron \& Marquart, 2011 : p.12).

Bref, toutes les relations de l'auteure-narratrice : gens, objets matériels ou culturels ont engendré la cristallisation de son moi autofictionnel, jouant sur la perception qu'elle veut donner au lecteur. Sur ce, arrivons au dixième et dernier point de la théorie de Gasparini. 


\section{0- La stratégie d'emprise du lecteur}

Grâce à son double pacte de lecture, pacte où la fiction et la réalité coexistent, l'autofiction arrive à capter l'attention du lecteur qui s'efforce tout au long du récit de rechercher des éléments textuels qui lui permettraient de démasquer l'auteur et de définir le genre littéraire du texte.

Doubrovsky (1988, p.69) affirme que l'auteur d'une autofiction devait "pour capter le lecteur rétif, lui refiler sa vie réelle sous les espèces plus prestigieuses d'une existence imaginaire ». À l'instar de Doubrovsky, Bouraoui implique le lecteur dès la première phrase de son récit : «Je viens vous voir parce que j'ai des mauvaises pensées " (MMP, p.9). Elle invite le lecteur-confident (le Docteur C.) à partager cette intimité, lorsqu'elle l'interpelle et l'installe dans la proximité et le relationnel. Le lecteur est intéressé à suivre l'auteure-narratrice dans sa quête laborieuse d'une vérité intime qu'elle s'acharne à déterrer des méandres de l'inconscient.

Bouraoui décrit ainsi son lecteur idéal, son lecteur modèle, comme "celui qui fait revivre le livre à travers ses yeux et sa pensée et qui arrive même à découvrir des choses auxquelles l'auteur n’a jamais pensé. Le lecteur peut réécrire un livre...Ce 
livre qui après n'appartient plus à l'auteur » (Bivona, 1994 : p.219).

En somme, le récit de "Mes Mauvaises Pensées", s'inscrivant à la frontière de genres, trouve sa place dans la constellation des écritures du moi. Une écriture aux confins de deux tendances contradictoires : l'autobiographie et la fiction donnant naissance à un "pacte oxymoronique » (Jaccomard, 1993 : p.81). Cette forme binaire «qui ne pouvait être dite et qui ne pouvait être tue» (Forest et Gaugain, $2001:$ p.172) coïncide avec Bouraoui. Cela veut dire que comme l'autofiction est un "genre" de l'entre-deux : entre le factuel et le fictionnel, entre l'autobiographique et le romanesque, entre le vécu et le fantasme, l'auteure-narratrice est elle-même une figure de l'entre-deux : entre deux cultures (algérienne et française), entre deux langues (le français et l'arabe), entre plusieurs espaces (l'Algérie, la France, la Suisse, etc.) mais surtout entre deux sexes (le féminin et le masculin). Elle confirme alors une écriture de mixité «où les droits de l'imaginaire sont au moins aussi forts que ceux du témoignage, où la fonction poétique submerge, travaille, transforme et révèle la fonction testimoniale » (ChauletAchour, 1998 : p.116). N'a-t-elle pas dit : « /l y a des auteurs qui masquent, d'autres qui ont choisi la vérité, moi je suis entre 


\section{deux» (MMP, p.70)?}

Ce récit remplit en réel la plupart des conditions de l'autofiction dégagées par Gasparini, ce qui engendre un travail d'élaboration littéraire approfondi qui permet à l'entre-deux/ l'entre-dire (l'autofiction) d'entrouvrir sur la vie de l'écrivaine une porte dérobée par où se glisse, en dehors de tout contrôle, son âme inconnue.

Selon nous, Bouraoui a utilisé des fragments de sa vie et de la vie des autres (la réalité) pour en faire un ensemble passionnant à lire (la fiction). D'autant plus, « l'autofiction ne permet pas au lecteur de disposer des clés pour différencier l'énoncé de réalité et l'énoncé de fiction » (Darrieussecq, 1996 : p. 377). Le lecteur se trouve ainsi pris dans un tourbillon de pensées introspectives, inscrites dans une construction textuelle constituée d'un seul long paragraphe. La phrase se déroule comme un engrenage d'association d'idées, de pensées et de sentiments puissants et perturbants, qui ne permet aucune pause. L'écrivaine se dévoile, revient sur un détail, l'abandonne pour le reprendre quelques pages plus loin, une écriture en boucle, en « spirale»(MMP, p.184), comme le souligne ellemême. 
Elle a atteint son idéal au goût des égarements à travers un texte autofictionnel dont le contenu narratif strictement intime est fictionnellement authentique et où elle ne cherche pas à choquer mais juste à révéler les battements de son cœur. Son désir prend les ailes d'une affirmation identitaire, lui permettant de se présenter comme elle est réellement et non pas comme d'autres voudraient la voir.

En conclusion, Nina Bouraoui concrétise l'écriture féminine contemporaine comme étant la revendication de la valorisation du corps et de l'inconscient tout en s'éloignant des mythes féminins élaborés par la littérature masculine. En ce sens, I'autofiction offre un lieu accueillant pour cette nouvelle image littéraire de la femme.

\section{Références bibliographiques}

- Benmahamed, A. (2000). L'écriture de Nina BOURAOUI : Éléments d'analyse à travers l'étude de cinq romans. Mémoire de Maîtrise. Université de Toulouse : Le Mirail. Récupéré de http:// www.limag.refer.org/Theses/BenmahamedMaitriseBouraoui.PDF

- Bivona, R. (1994). "Entrevue avec Nina Bouraoui », annexe à la thèse de doctorat : Nina Bouraoui, un sintomo di letteratura migrante nell'area franco-magrebina. Doctorat. Université de Palerme: G.S. SANTANGELO \& A.-M. RUBINO. Récupéré de http ://www.limag. refer.org/Theses /Bivona .pdf

- Blanchot, M. (1959). Le livre à venir. Paris : Gallimard. 
- Bouraoui, N. (2005). Mes Mauvaises Pensées. Paris : Stock.

- Bouraoui, N. (2000). Garçon manqué. Paris : Stock.

- Burgelin, C. (2010). Modiano et ses "je". Dans C. Burgelin, I. Grell et R-Y. Roche (dir.). Autofiction(s) : colloque de Cerisy (p. 207-222). Lyon : PUL.

- Burgelin, C., Grell, I. et Roche, R-Y. (2010). Autofiction(s), actes du colloque de Cerisy-la-Salle en été 2008 (p. 461- 482). Lyon : PUL.

- Caron, D. \& Marquart, S. (2011). Les Revenantes : Charlotte Delbo, la voix d'une communauté à jamais déportée. Toulouse: Presses universitaires du Mirail.

- Chaulet-Achour, C. (1998). Noûn. Algériennes dans l'écriture. Biarritz : Atlantica.

- Chevalier, J. \& Gheerbrant, A. (1986). Dictionnaire des symboles. Paris : Robert Laffont / Jupiter.

- Colonna, V. (2004). Autofiction et autres mythomanies littéraires. Auch : Éditions Tristram.

- Colonna, V. (1989). L'autofiction : essai sur la fictionnalisation de soi en littérature. Thèse de doctorat, sous la direction de Gérard Genette, E.H.E.S.S. Récupéré de http://tel.archives-ouvertes.fr /docs/00/04/70/ 04/ PDF/tel-00006609.pdf/

- Darrieussecq, M. (1996). L'autofiction, un genre pas sérieux. Poétique, 107, (septembre), 369-380.

- Doubrovsky, S. (1993). Textes en main. Dans S. Doubrovsky, J. Lecarme et Ph. Lejeune (dir.). Autofictions \& Cie, (pp. 207-217). RITM, 6 : Université de Paris $X$. 
- Doubrovsky, S. (1988). Autobiographiques : De Corneille à Sartre. Paris : PUF.

- Doubrovsky, S. (1977). Fils. Paris : Galilée.

- Doubrovsky, S. \& Contat, M. (2001). Quand je n'écris pas, je ne suis pas écrivain. Entretien avec M. Contat. Genesis, 16 (1), 119-135.

- Forest, Ph. \& Gaugain, C. (2001). Les romans du je. Nantes : Pleins Feux.

- Gasparini, Ph. (2008). Autofiction. Une aventure du langage. Paris : Seuil.

- Gasparini, Ph. (9 octobre 2009). De quoi l'autofiction est-elle le nom ? Dans Conférence prononcée à l'Université de Lausanne. Récupéré de http://www.autofiction.org/index.php?post/2010/ 01/ 02/De-quoiI-autofiction-est-elle-le-nom-Par-Philippe-Gasparini.

- Genette, G. (1991). Fiction et diction. Paris : Seuil.

- Giesbert, F-O. (08/10/2005). Bouraoui Nina, Culture et Dépendance, émission télévisée. Récupéré de https://devideows.ga/movies/download-free-culture-etd\%C3\%A9 pendances-faut-il-vraiment-\%C3\%AAtre-moderne-web-dl.html.

- Hubier, S. (2003). Littératures intimes : les expressions du moi, de l'autobiographie à l'autofiction. Paris : Armand Colin.

- -Jaccomard, H. (1993). Lecteur et lecture dans l'autobiographie française contemporaine : «Serge Doubrovsky : Le pacte oxymoronique ». Genève : Droz.

- Jay, S. (19 mai 2010). Nina Bouraoui et Mina Simpson : Du talent pour se chercher. Le Soir Échos. Récupéré de http://www.lesoir- 
echos.com/2010/05/19/salim-jay-nina-bouraoui-et-mina-simpson

$\% \mathrm{C} 2 \% \mathrm{~A} 0$-du-talent-pour-se-chercher/

- Jenny, L. (2003). L'autofiction. Méthodes et problèmes, Genève : Département de français moderne. Récupéré de https://www.unige .ch/lettres/framo/enseignements/methodes/autofiction/afintegr.html.

- Journet, A. (9 mai 2011). Nina Bouraoui et Sara Stridsberg : L'écrivain est un marginal. L'Express. Récupéré de http://www.lexpress.fr/culture/livre/nina-bouraoui-et-sara-stridsberg l-ecrivain-est-un-marginal_990537.html.

- Laflamme, E. (2006). II faut déconstruire avant de construire / Mes Mauvaises Pensées de Nina Bouraoui, Éditions Stock, 286 p. Dans Spirale : Arts • Lettres • Sciences humaines, 209, 48-49. Récupéré de https://www.erudit.org/fr/revues/spirale/2006-n209spirale10598 31/17626ac/.

- Lejeune, Ph. (1995). Le pacte autobiographique. Paris : Seuil.

- Lejeune, Ph. (1986). Moi aussi. Paris : Seuil.

- Mijolla, A. de. (2005). Dictionnaire international de la psychanalyse. Paris : Hachette littératures.

- Pons, G. (1999). Le paysage : sauvegarde et création. Seyssel : Champ Vallon.

- Richard, A. (2013). L'autofiction et les femmes : Un chemin vers l'altruisme?. Paris : L'Harmattan.

- Ricœur, P. (1990). Soi-même comme un autre. Paris : Le Seuil.

- Robin, R. (2002). Autobiographie et judéité chez Jacques Derrida. Études françaises, 38 (1-2), 207-218. 
- Robin, R. (1997). Le Golem de l'écriture. De l'autofiction au Cybersoi. Montréal : $X \ddot{Y} Z$ éditeur.

- Viart, D. (1997). Une mémoire inquiète. La Route des Flandres de Claude Simon. Paris : PUF.

- Vilain, Ph. (2005). Défense de Narcisse. Paris : Grasset. 